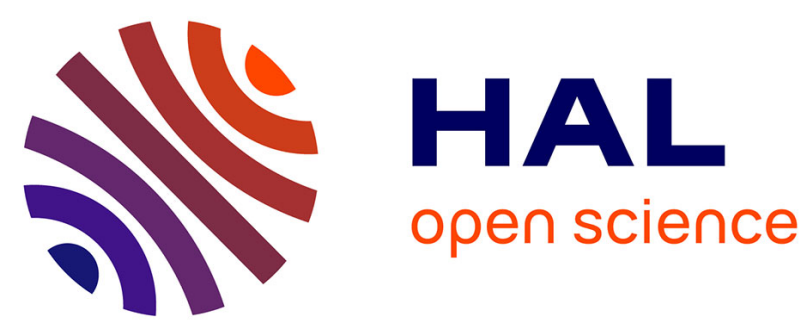

\title{
Analytical evaluation of point of care cTnT and clinical performances in an unselected population as compared with central laboratory highly sensitive cTnT
}

Anne Marie Dupuy, Mustapha Sebbane, François Roubille, Thibault Coste, Anne-Sophie Bargnoux, Stéphanie Badiou, Nils Kuster, Jean-Paul Cristol

\section{To cite this version:}

Anne Marie Dupuy, Mustapha Sebbane, François Roubille, Thibault Coste, Anne-Sophie Bargnoux, et al.. Analytical evaluation of point of care cTnT and clinical performances in an unselected population as compared with central laboratory highly sensitive cTnT. Clinical Biochemistry, 2015, 48 (4-5), pp.334 - 339. 10.1016/j.clinbiochem.2014.09.027 . hal-01756121

\section{HAL Id: hal-01756121 \\ https://hal.umontpellier.fr/hal-01756121}

Submitted on 14 Sep 2018

HAL is a multi-disciplinary open access archive for the deposit and dissemination of scientific research documents, whether they are published or not. The documents may come from teaching and research institutions in France or abroad, or from public or private research centers.
L'archive ouverte pluridisciplinaire HAL, est destinée au dépôt et à la diffusion de documents scientifiques de niveau recherche, publiés ou non, émanant des établissements d'enseignement et de recherche français ou étrangers, des laboratoires publics ou privés. 


\title{
Analytical evaluation of point of care cTnT and clinical performances in an unselected population as compared with central laboratory highly sensitive cTnT
}

\author{
Anne Marie Dupuy ${ }^{a}$, Mustapha Sebbane ${ }^{b}$, François Roubille ${ }^{c}$, Thibault Coste ${ }^{a}$, Anne Sophie Bargnoux ${ }^{a}$, \\ Stéphanie Badiou ${ }^{a}$, Nils Kuster ${ }^{\mathrm{a}}$, Jean Paul Cristol ${ }^{\mathrm{a}, *}$ \\ a Biochemistry Laboratory, Lapeyronie Hospital, Montpellier, France \\ ${ }^{\mathrm{b}}$ Emergency Department, Lapeyronie Hospital, Montpellier, France \\ c Cardiology Department, Arnaud de Villeneuve Hospital, Montpellier, France
}

\section{A B S T R A C T}

Objectives: To report the analytical performances of the Radiometer AQT90 FLEX® cTnT assay (Neuilly-Plaisance, France) and to evaluate the concordance with hs-cTnT results from central laboratory for the diagnosis of acute myocardial infarction (AMI) at baseline and during a short follow-up among unselected patients admit-ted in emergency room or cardiology department.

Design and methods: Analytical performances of AQT90 FLEX® ${ }^{\circledR}$ TnT immunoassay included imprecision study with determination of a coefficient of variation at $10 \%$ and $20 \%$, linearity, and limit of detection. The concordance study was based on samples obtained from 170 consecutive patients with chest pain suggestive of acute coronary syndrome (ACS) admitted in the emergency room or cardiology department. The kinetic study (within 62 additional samples $3 \mathrm{~h}$ later) was based on absolute delta criterion and the combination of relative change of 30\% with absolute change of $7 \mathrm{ng} / \mathrm{L}$.

Results: The cTnT assay from Radiometer was evaluated as clinically usable, although less sensitive than the Roche hs-cTnT assay as demonstrated by the concordance and the kinetic studies.

Conclusions: In non-selected population, the CTnT AQT Flex@ assay on AQT90@ with kinetic change at $3 \mathrm{~h}$, provides similar clinical classification of patients, particularly for AMI group as compared to central laboratory hs-cTnT assay and could be suitable for clinical use.

Keywords:

Point of care

Highly sensitive cardiac troponin

Kinetic change

Diagnostic performance

\section{Introduction}

Point of care (POC) cardiac markers testing offers potential benefits from a clinically oriented point of view regarding cardiology, especially when central laboratory remains unable to meet the 1-h turnaround time (TAT) for the measurement of cardiac troponin (cTn). In this case, the National Academy of Clinical Biochemistry (NACB) guidelines as well as the European Society of Cardiology recommend the implementation of POC system [1,2]. Different POC devices for cTnT or I are available and theoretically the sensitivity of the assay system should not differ from that provided by the central laboratory system. However, evaluating a given POC methodology compared to a central laboratory assay remains mandatory, as recently suggested in studies on this topic $[3,4]$. Clearly, the lack of standardization and harmonization within cTn assays including different cut-off levels regarding cTnT an cTnI could be misleading, even with the implementation of highly

\footnotetext{
* Corresponding author at: 371, avenue du doyen Gaston Giraud, 34295 Montpellier Cédex 5, France. Fax: + 33467338393.

E-mail address: jp-cristol@chu-montpellier.fr (J.P. Cristol).
}

sensitive assays (hs-cTn). There are only two systems measuring cTnT, and at least seven devices measuring CTnI. The POC devices should also meet the consensus diagnostic threshold for acute myocardial infarction (AMI), with a coefficient of variation $<10 \%(10 \% \mathrm{CV})$ or less at the 99 th percentile. However, a CV at 99 th percentile of $10 \%$ to $20 \%$, according to Apple et al. [5] does not lead to significant patient misclassification when interpreting serial cTn results [6] and the cTn assay was considered as "clinically usable". If the CV at 99th percentile is greater than $20 \%$, the routine use of cardiac troponin assays is not acceptable. As results provided by biological platforms are delayed, the implementation of POC device is growing worldwide. To improve the specificity of hs-cTn for the diagnosis of AMI, kinetic studies based on relative or absolute delta changes have been proposed. However, using relative or absolute changes remains debated as well as the magnitude of the kinetic change to discriminate between acute and chronic increases. The best option could be the absolute change allowing the accuracy for AMI compared to the relative change $[7,8]$ and the combination of the relative and absolute changes [9].

Here, we report the analytical performances of the Radiometer AQT90 FLEX® ${ }^{\circledR T n T}$ assay (Neuilly-Plaisance, France) and we evaluate 
the concordance with hs-cTnT results from central laboratory in diagnosing AMI at baseline and during a kinetic study. The main aim was to compare these two methods among unselected patients admitted in both emergency and cardiology departments.

\section{Materials and methods}

\section{cTnT POC method}

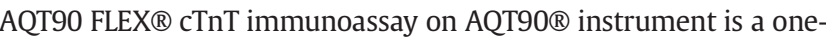
step sandwich immunofluorometric assay [10]. The minimum sample volume is $2 \mathrm{~mL}$ (independent of the number of AQT90 FLEX® tests requested). EDTA or lithium heparinized whole blood or plasma samples are appropriate. The 99th percentile cTnT reference value for the AQT90 FLEX ${ }^{\circledR}$ was defined as $17 \mathrm{ng} / \mathrm{L}[11]$, the $10 \% \mathrm{CV}$ and the lowest level of detection were claimed by the manufacturer at 30 and $10 \mathrm{ng} / \mathrm{L}$, respectively.

\section{cTnT central laboratory method}

At central laboratory, hs-cTnT was measured using a fifthgeneration assay from Roche on Cobas8000/e602® instrument (Roche Diagnostics, Mannheim, Germany) previously described [12]. The 99th percentile cut-off point of the hs-cTnT assay among healthy individuals has been described at $14 \mathrm{ng} / \mathrm{L}$, and the lowest concentration measurable with a coefficient of variation $<10 \%(10 \% \mathrm{CV})$ is $13 \mathrm{ng} / \mathrm{L}$ [13]. The lowest level of detection of this assay is $5 \mathrm{ng} / \mathrm{L}$.

\section{Analytical performances of AQT90 FLEX® CTnT immunoassay}

Imprecision studies were based on the CLSI EP5 protocol (duplicate measurements twice per day on 4 levels for 20 days) [14] from plasma pool. A plasma pool with cTnT concentration of $140 \mathrm{ng} / \mathrm{L}$ was used to test the linearity. The pool was diluted at the following final concentrations: 93, 70, 46, 35, 28, 17 and $14 \mathrm{ng} / \mathrm{L}$. Each concentration was measured in duplicate. The limit of detection (LoD) was determined according to the current CLSI standard [15] from plasma samples. The LoD provided by the manufacturer is $10 \mathrm{ng} / \mathrm{L}$. Both samples with cTnT below $10 \mathrm{ng} / \mathrm{L}$ (Sample A) and low concentration samples with approximates 3 and 4 times the assay's sensitivity claimed by the manufacturer (Sample B) were tested. For all selected assays, 10 replicates of the A sample and 10 replicates of both low concentration samples $(3 \times$ and $4 \times$ ) were run. The LoD was calculated as LoD $=$ LoA +1.645 oS, where oS was the standard deviation of the population of the low concentration sample measurements. The concordance study was based on samples obtained from 170 consecutive patients with chest pain suggestive of acute coronary syndrome (ACS) admitted to the emergency room and the cardiology departments of Lapeyronie and Arnaud de Villeneuve university hospitals respectively (Montpellier, France). Testing was performed simultaneously measuring the hs-cTnT on the Cobas8000/e602 ${ }^{\circledR}$ module and the cTnT on the AQT90 FLEX $®$ instrument. The 170 samples at baseline were used to compare studies between the two methods. Only hs-cTnT values were used for the clinical diagnosis.

\section{Clinical studies}

Kinetic studies were performed only if the clinical diagnosis and/or the therapeutic option were not determined in the first hour of hospitalization. Thus, among the 170 patients included, only 62 additional samples could be obtained $3 \mathrm{~h}$ later. The clinical records of all patients were evaluated to confirm the diagnosis following the enrollment. The final diagnoses were adjudicated using the hs-cTnT from Roche by a senior emergency physician and by a cardiologist. Patients were divided into 2 diagnostic groups. Diagnosis at discharge was AMI or non-AMI. Diagnosis of AMI was established according to the universal definition of myocardial infarction [16]: patients with symptoms of cardiac ischemia with simultaneous ECG changes and/or increased cardiac markers such as troponin, were categorized as having AMI and further subcategorized into ST-segment elevation myocardial infarction (STEMI) and non-STsegment elevation myocardial infarction (NSTEMI) [16]. STEMI was defined by the persistent elevation of the segment ST of $\geq 1 \mathrm{~mm}$ in 2 contiguous electrocardiographic leads or by the presence of a new left bundle branch block with positive cardiac enzyme results. NSTEMI was defined as the occurrence of acute myocardial infarction in the setting of positive cardiac marker results with or without accompanying electrocardiographic changes other than ST segment elevation. Unstable angina (UA) was diagnosed if the ECG was uncertain and with negative cTn levels. Upon admission, patients with UA or NSTEMI may not have been distinguished and the diagnosis of NSTEMI was established according to cardiac biomarkers. All patients with excluded AMI (nonAMI) were categorized to a number of clinical conditions cardiac, noncardiac or unknown origin (UO). Cardiac causes included heart failure, UA, arrhythmia, TakoTsubo, invasive procedure and non-specific chest pain (NSCP). Non-cardiac causes included pulmonary disease, stroke, syncope, renal disease, infection and symptoms of unknown origin (UO).

All investigations were performed in accordance with the principles of the Declaration of Helsinki and approved by the local ethics committee. Informed consent was obtained from patients prior to their participation in the study.

\section{Statistical analysis}

The 99th percentile (14 ng/L) cut point for hs-cTnT assay from Roche was used. For cTnT from Radiometer we used the cut-off value $20 \%$ CV (17 ng/L) as recommended for POC instrument by Jaffé et al. [17] and corresponding to the 99th percentile claimed by the manufacturer. For correlations between results obtained with different assays, linear regression analysis and Bland-Altman plots were performed. Receiver operator characteristic (ROC) curves are presented for the diagnosis of AMI and 95\% CI around the AUC was calculated. An absolute delta change was calculated between highest cTnT concentration from $3 \mathrm{~h}$ sample and baseline ( $\mathrm{Cmax}$ ( $3 \mathrm{~h}$ or $6 \mathrm{~h}$ ) - Cbaseline) expressed in nanograms per liter. Negative changes indicated a fall whereas positive changes marked a rise. A significant rise or fall was when there was at least one value above the 99th percentile or above the $20 \% \mathrm{CV}$ with a magnitude of change of $7 \mathrm{ng} / \mathrm{L}$. In addition, we considered the combination of relative change of $30 \%$ with absolute change of $7 \mathrm{ng} / \mathrm{L}$ reported by Irfan et al. [9]. In calculation of the delta change, for patients with value below the LoD, we took the absolute value of $10 \mathrm{ng} / \mathrm{L}$ and $5 \mathrm{ng} / \mathrm{L}$ for Radiometer cTnT and Roche hs-cTnT, respectively. Data were compared using Student's t-test or Mann-Whitney test for continuous variables, and the Fisher's exact test for differences in frequencies. A $p$ value $<0.05$ was considered as statistically significant.

\section{Results}

\section{Analytical performances of AQT90 FLEX® cTnT assay}

All results are presented in Table 1 . Based on a $19.3 \%$ CV at 99th percentile (17 ng/L) the AQT90 FLEX® cTnT assay was confirmed as "clinically usable". Functional sensitivity defined as the smallest cTnT level that could be measured with a CV of 10\%, was $30 \mathrm{ng} / \mathrm{L}$ similar to the description provided by the manufacturer. The linear equation of linearity was Radiometer $=1.03$ Roche $-10.64, r^{2}=0.98$, with a mean recovery (SD) percentage of $80 \%$ (10\%). The LoD in our condition was $15.4 \mathrm{ng} / \mathrm{L}$.

Fig. 1 shows the Passing-Bablok regression analysis of the 170 values of cTnT as a function of the assays. We observed two distinct peaks according to the chosen assay, at $10 \mathrm{ng} / \mathrm{L}$ and at $17-50 \mathrm{ng} / \mathrm{L}$. Indeed, a large proportion of patients (35\%) had cTnT values $<10 \mathrm{ng} / \mathrm{L}$ with 
Table 1

Analytical characteristics of AQT90 FLEX® CTnT assay on AQT90® instrument from Radiometer.

\begin{tabular}{llll}
\hline Total CV imprecision results & Mean (ng/L) & SD (ng/L) & CV (\%) \\
\hline Plasma Pool 1 & 17 & 3.26 & 19.20 \\
Plasma Pool 1 & 27 & 3.11 & 11.53 \\
Plasma Pool 2 & 33 & 2.94 & 8.81 \\
Plasma Pool 3 & 51 & 3.90 & 7.60 \\
Limit of detection (ng/L) & 15.40 & & \\
Linearity & & & \\
Mean (SD) recovery, \% & $80(10)$ & & \\
\hline
\end{tabular}

$\mathrm{CV}$, coefficient of variation; SD, standard deviation.

Radiometer assay, against only $5 \%$ of patients with Roche hs-cTnT. About $50 \%$ of patients had Roche hs-cTnT values ranging from 17 to $50 \mathrm{ng} / \mathrm{L}$, against 25\% with the Radiometer cTnT assay. From $90 \mathrm{ng} / \mathrm{L}$ the percentage of patients was close between both assays. The part $\mathrm{B}$ of the Fig. 1 shows a Bland-Altman plots that indicates a systematic bias between the assays.

The concordance was established between hs-cTnT from Roche cutoff of $14 \mathrm{ng} / \mathrm{L}$ and cTnT assay from Radiometer cut-off of $17 \mathrm{ng} / \mathrm{L}$ (20\% $\mathrm{CV}$ ). Our results indicated $58.7 \%$ of concordance between the two methods. A large number of subjects were discordant with $70 \%$ (41.2\%) presenting hs-cTnT Roche values $>14 \mathrm{ng} / \mathrm{L}$ and cTnT Radiometer values $<17 \mathrm{ng} / \mathrm{L}$. No subjects showed hs-cTnT Roche $<14 \mathrm{ng} / \mathrm{L}$ associated with cTnT Radiometer $>17 \mathrm{ng} / \mathrm{L}$. Thus, a large proportion of patients had results above the decision limit when the central laboratory assay was applied, but below the decision limit of the POC system.

\section{Baseline levels according to the final diagnosis}

After initial clinical and ECG evaluation, 108 patients were discharged of our study. In the remaining 62 patients a short kinetic analysis was performed within $3 \mathrm{~h}$ after admission. In case of uncertain diagnosis additional samples were obtained $6 \mathrm{~h}$ later. The final adjudicated clinical diagnoses were categorized to AMI including STEMI $(n=4)$, NSTEMI $(n=4)$, non-AMI $(n=48)$ and UO $(n=6)$. Subcategories were cardiac causes including heart failure $(n=8)$, UA $(n=2)$, arrhythmia $(n=5)$, TakoTsubo $(n=1)$, invasive procedure $(n=6)$ and $\operatorname{NSCP}(n=7)$. Non-cardiac causes are composed of pulmonary disease as pulmonary embolism and acute respiratory distress syndrome $(n=7)$, stroke $(n=5)$, syncope $(n=5)$, renal disease $(n=1)$, and infection $(n=1)$. The baseline demographic characteristics of the 62 patients included in the study (56.4\% male, median age \pm SD: 71 years \pm 16) subdivided by final diagnoses are described in Table 2 . The median cTnT concentrations at admission were significantly higher with Roche (27 ng/L, IQR, 15-67) than with Radiometer (10 ng/L, IQR, 10-34) $(p<0.001)$. The proportions of Roche hs-cTnT results above the given cut-off were significantly higher than that of the Radiometer cTnT assay for all the clinical categories.

Fig. 2 shows the scattergram of cTnT results according to the assays and the two main categories AMI vs. non-AMI. The baseline levels with both assays were significantly higher in patients with AMI $(p<0.001)$. In AMI group, at presentation $8(100 \%)$ patients had hs-cTnT Roche above the 99th percentile, and 6 (75\%) patients had cTnT Radiometer above $17 \mathrm{ng} / \mathrm{L}$. For both assays, the percentage of the distribution between AMI vs. non-AMI was statistically different $(p<0.001)$. The clinical performances of the assays were further evaluated by ROC analysis. As shown in Fig. 2 (part B) the AUCs of the ROC curves were not significantly different between central laboratory assay and Radiometer assay.

\section{Kinetic analysis}

Our kinetic study was based on absolute delta criterion and the combination of relative change of $30 \%$ with absolute change of $7 \mathrm{ng} / \mathrm{L}$.
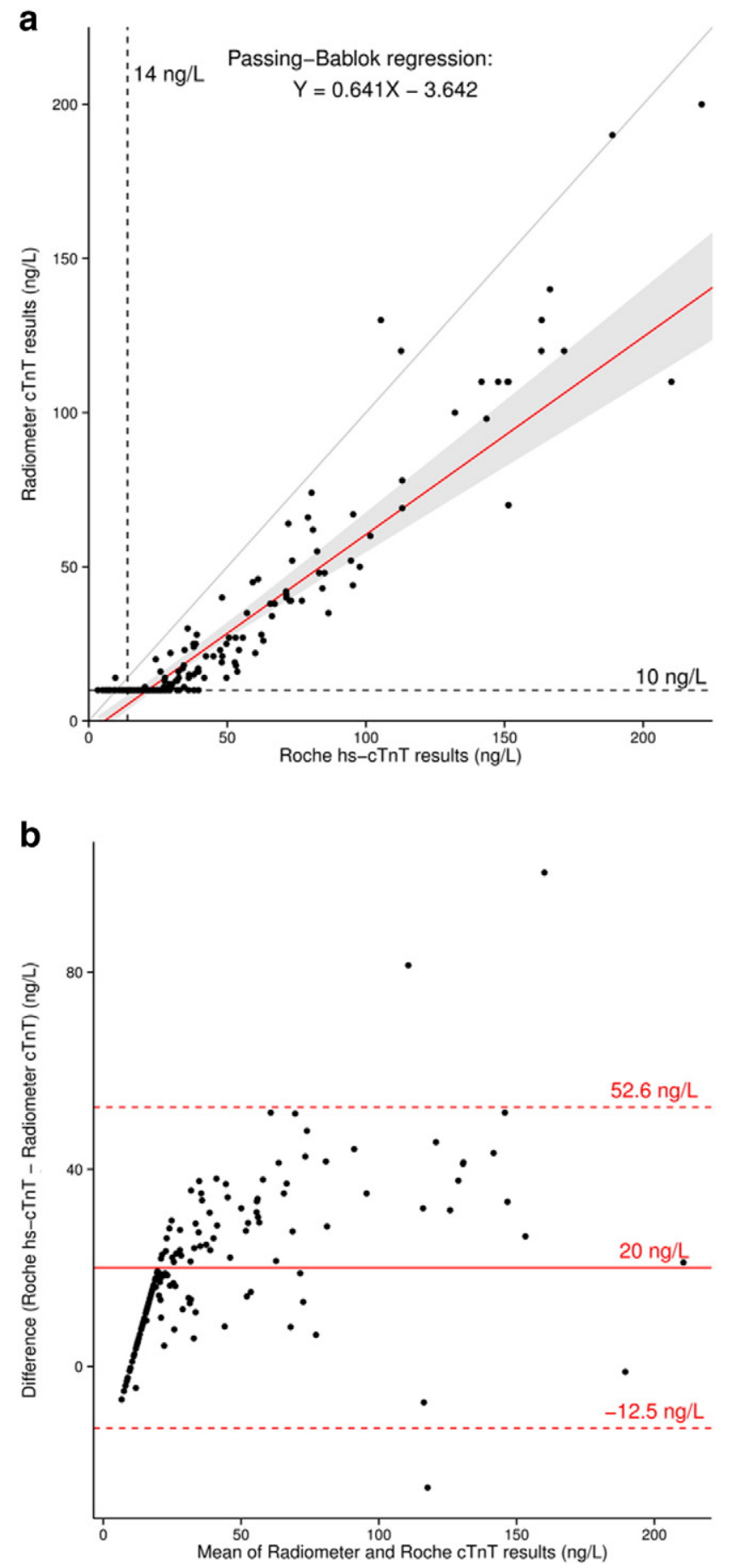

Fig. 1. (a) Passing-Bablok regression analysis of the Roche hs-cTnT vs. Radiometer cTnT assays on the total population $(n=170)$ and (b) Bland-Altman bias plot. Dotted horizontal lines indicate two standard deviations around the difference of the two methods. Troponin values above $220 \mathrm{ng} / \mathrm{L}$ were excluded from the analysis.

In the AMI group, an absolute change $>7 \mathrm{ng} / \mathrm{L}$, even more $>14 \mathrm{ng} / \mathrm{L}$ was observed in $100 \%$ of patients with both assays. In non-AMI group, all patients $(n=54)$ had at least one value above the 99th percentile with hs-cTnT Roche, whereas 50\% $(n=27)$ of patients had at least one value above $17 \mathrm{ng} / \mathrm{L}$ with cTnT Radiometer. A delta absolute change above $7 \mathrm{ng} / \mathrm{L}$ in the non-AMI group was found in $72 \% \mathrm{vs}$. 37\% with Roche hs-cTnT vs. Radiometer cTnT assay, respectively $(p<0.001)$. In both assays, absolute changes were higher in patients with baseline cTnT levels above their respective cut-off. 
Table 2

Characteristics at admission of patients having kinetic of troponin $(n=62)$.

\begin{tabular}{|c|c|c|c|c|}
\hline & All & Hs-cTnT above the cut-off $14 \mathrm{ng} / \mathrm{L}, n(\%)$ & cTnT above the cut-off $17 \mathrm{ng} / \mathrm{L}, n(\%)$ & $p$ \\
\hline Number, $n(\%)$ & 62 & $53(85)$ & $23(37)$ & $<0.001$ \\
\hline Age, mean (range), years & $71(93-22)$ & $72(90-22)$ & $68(90-36)$ & NS \\
\hline Male sex, $n(\%)$ & $35(56)$ & $29(83)$ & $12(34)$ & NS \\
\hline Discharge diagnosis (main categories) & $\mathrm{n}$ & & & \\
\hline AMI & 8 & $8(100)$ & $6(75)$ & NS \\
\hline Non-AMI & 54 & $45(83)$ & $18(33)$ & $<0.001$ \\
\hline Discharge diagnosis for non-AMI (subcategories) & $n$ & & & \\
\hline Cardiac & 29 & $22(76)$ & $9(31)$ & $<0.001$ \\
\hline Heart failure & 8 & $6(75)$ & $4(50)$ & \\
\hline UA & 2 & $2(100)$ & $1(50)$ & \\
\hline Dysrythmia & 5 & $4(80)$ & $1(20)$ & \\
\hline TakoTsubo & 1 & $1(100)$ & $1(100)$ & \\
\hline Invasive procedure & 6 & $5(83.3)$ & 0 & \\
\hline NSCP & 7 & $4(57)$ & $2(29)$ & \\
\hline Non-cardiac pulmonary & 19 & $18(95)$ & $7(37)$ & $<0.001$ \\
\hline $\mathrm{PE}$ & 3 & $3(100)$ & $2(67)$ & \\
\hline ARDS & 4 & $4(100)$ & $1(25)$ & \\
\hline Stroke & 5 & $5(100)$ & $2(40)$ & \\
\hline Syncope & 5 & $4(80)$ & $1(20)$ & \\
\hline Renal & 1 & $1(100)$ & 0 & \\
\hline Infectious & 1 & $1(100)$ & $1(100)$ & \\
\hline UO & 6 & $5(83)$ & $2(33)$ & NS \\
\hline
\end{tabular}

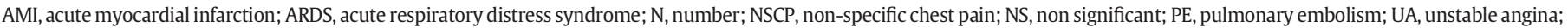
UO, unknown origin.

The proportion of patients according to the combination of the amount of relative, absolute changes of hs-cTnT Roche or cTnT Radiometer to AMI or other diagnosis categories is shown in Fig. 3. All the AMI patients fulfilled the $30 \%$ and the $7 \mathrm{ng} / \mathrm{L}$ delta change criterion whatever the assays. In the non-AMI group, only $35 \%$ of patients had a relative change above $30 \%$ and absolute change above $7 \mathrm{ng} / \mathrm{L}$ with Radiometer cTnT using cut-off of $17 \mathrm{ng} / \mathrm{L}$, in contrast with the Roche hs-cTnT, the percentage reached 55\% $(p<0.001)$. With Roche hs-cTnT in the subgroup of patients classified in cardiac causes, the percentage having relative change $>30 \%$ and absolute change $>7 \mathrm{ng} / \mathrm{L}$ was twice higher than with Radiometer cTnT. The lowest percentage for patients classified in the subgroup of non-cardiac causes was obtained with Radiometer cTnT $20 \%$ vs. $26 \%$ using the Roche hs-cTnT.

In Table 3, the magnitude of kinetic changes for each clinical category is reported. The absolute changes with Roche hs-cTnT or Radiometer cTnT levels at presentation and after $3 \mathrm{~h}$, were significantly higher in patients with AMI ( $p<0.01$ for both assays). In patients with AMI, the differences between the absolute change with hs-cTnT Roche or cTnT Radiometer were not significant. Similar results were found in non-

\section{a}

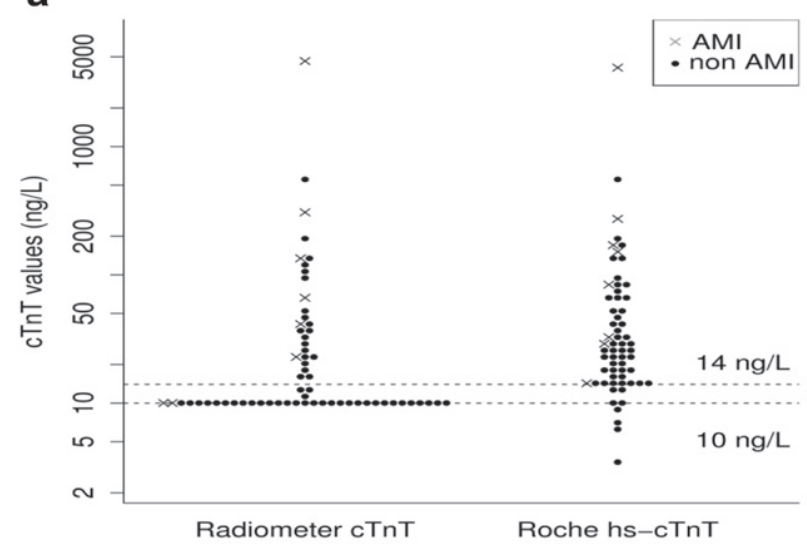

AMI group. The magnitude of the delta change (median, IQR) demonstrated a better performance of absolute delta change in comparison with relative change (data not shown) because there was no overlap between AMI and non-AMI patients.

The magnitudes of rising Radiometer cTnT concentrations were higher than with Roche hs-cTnT for absolute cTnT changes as well as among AMI patients or patients with other diagnoses. Rising values were found more frequently as compared to falling values. Conversely, falling values were noted more often ( 6 patients) when cTnT Radiometer was used, as compared to patients ( 4 patients) with hs-cTnT Roche determination. The very low number (one case by category) of falling results requires caution in the interpretation.

\section{Discussion}

The main aim of our study was to compare the use of POC methodology to cTnT central laboratory method among unselected patients, especially the impact of highly sensitive cTnT vs. less sensitive POC assays in the management of patients with suspected AMI. The main finding

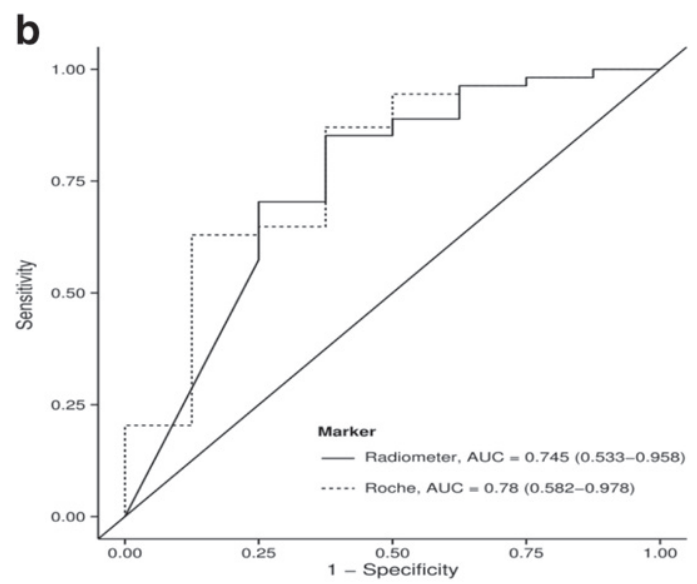

AMI, acute myocardial infarction

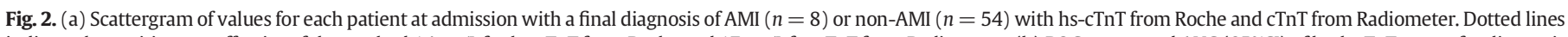

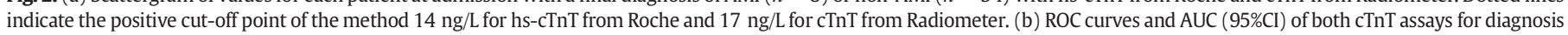
performances. AMI, acute myocardial infarction. 


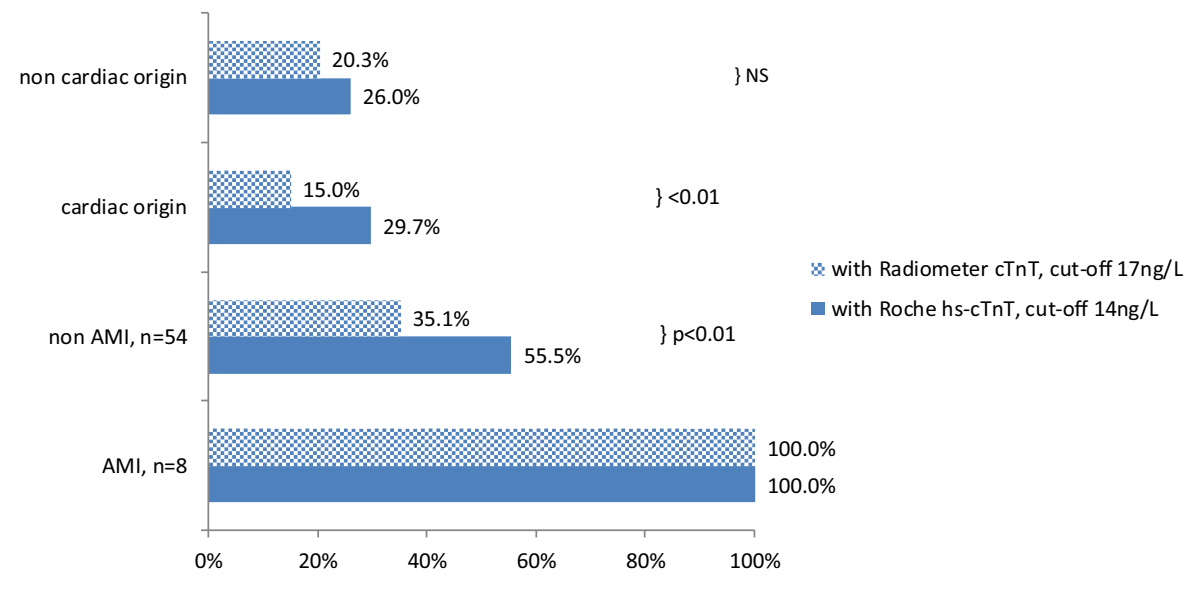

AMI, acute myocardial infarction; NS, not significant

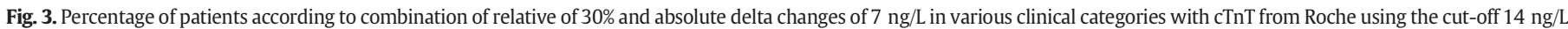
and from Radiometer using the $20 \%$ CV (17 ng/L) cut-off. AMI, acute myocardial infarction; NS, not significant.

was that using clinically available POC cTn assay for kinetic analysis did not result in false-negative results. Secondly, we showed that (1) the increased sensitivity of cTnT assays was detrimental to the specificity (2) challenging the thresholds of absolute delta change at $7 \mathrm{ng} / \mathrm{L}$ reported by Reichlin et al. [7] and (3) these results underlined the benefit of the combination of the relative and absolute delta change corroborating Irfan et al. [9].

Our analytical evaluation confirmed the status of the cTnT from Radiometer as clinically usable according to the proposed scorecard by Apple [5]. Indeed the total imprecision at the 99th percentile was found close to $20 \%$. This threshold could be used in clinical practice according to Jaffé et al. [17], on the one hand, because the increments of imprecision from $5 \%$ to $20 \%$ did not alter the specificity/sensitivity [17] and on the other hand, a $10 \% \mathrm{CV}$ could falsely limit sensitivity. Indeed, using the $10 \% \mathrm{CV}$ (30 ng/L) with the POC instrument, resulted in an increase of $57 \%$ of values below the cut-off by comparison of $10 \%$ CV hscTnT (14 ng/L) (data not shown). The observed LOD was $15.4 \mathrm{ng} / \mathrm{L}$, higher than the value provided by the manufacturer. It could be suggested that this discrepancy was due to the use of a patient sample (with an undetectable troponin level determined with Radiometer) rather than sample without analyte as zero standard. The linearity of the POC method was acceptable and confirmed the manufacturer's data.

Comparing Roche hs-cTnT values with Radiometer cTnT values several observations can be drawn. Firstly, the hs-cTnT method detected more samples with small level of cTnT than with the cTnT from the Radiometer method. Secondly, even if both methods recognize the same antigens, the cTnT levels were not directly comparable. Indeed, we found discrepant 70 samples which displayed hs-cTnT values

Table 3

Median absolute kinetic changes of positive and negative kinetic patterns subdivided by diagnosis categories with hs-cTnT from Roche and cTnT from Radiometer.

\begin{tabular}{|c|c|c|c|c|}
\hline \multirow{3}{*}{ Kinetic changes } & \multicolumn{2}{|c|}{$\begin{array}{l}\text { Hs-cTnT from Roche } \\
\text { (cut-off } 14 \mathrm{ng} / \mathrm{L} \text { ) }\end{array}$} & \multicolumn{2}{|c|}{$\begin{array}{l}\text { cTnT from Radiometer } \\
\text { (cut-off } 17 \mathrm{ng} / \mathrm{L} \text { ) }\end{array}$} \\
\hline & \multicolumn{2}{|c|}{ Absolute kinetic changes, ng/L } & \multicolumn{2}{|c|}{ Absolute kinetic changes, ng/L } \\
\hline & Rising & Falling & Rising & Falling \\
\hline AMI, $n=8$ & $70(54-177)$ & - & $76(34-244)$ & $10^{\mathrm{a}}$ \\
\hline Non-AMI, $n=54$ & $13(7-31)$ & $27(27-28)$ & $18(9-30)$ & $41(59-22)$ \\
\hline Cardiac & $12(6-32)$ & $28^{a}$ & $20(12-46)$ & $32^{\mathrm{a}}$ \\
\hline Non-cardiac & $17(7-32)$ & $26^{\mathrm{a}}$ & $15(7-23)$ & $42(60-25)$ \\
\hline UO & $23(19-32)$ & - & $15(10-22)$ & $4^{a}$ \\
\hline
\end{tabular}

Values are median (interquartile range).

AMI, acute myocardial infarction; N, number; UO, unknown origin.

a $n=1$.
$>14 \mathrm{ng} / \mathrm{L}$ associated with Radiometer cTnT values $<17 \mathrm{ng} / \mathrm{L}$ (median of values $26 \mathrm{ng} / \mathrm{L}$ with hs-cTnT vs. $10 \mathrm{ng} / \mathrm{L}$ with Radiometer cTnT). These data were consistent with the increased sensitivity provided by the hs-cTnT assay from Roche.

In the non-AMI group at admission only $15 \%$ of subjects had hs-cTnT values below 99th percentile whereas with the cTnT from Radiometer the percentage rises $69 \%$. The high percentage of values above the 99th percentile in an unselected population could be misleading as regards the interpretation of the values of the hs-cTnT for diagnosis of AMI. In our study, 2 patients with a final diagnostic of AMI were not detected using the POC assay at baseline by contrast with the central laboratory high-sensitivity assay. Importantly the ability to identify patients quickly could facilitate rapid triage and better management. However, this increased sensitivity at baseline raises the problem for non-AMI patients who presented elevated cTn. Indeed we detect patients with high cTn values in the AMI as well as in the non-AMI group underlining the lack of specificity. At baseline, an increase in specificity was at the expense of sensitivity and conversely the increase of sensitivity at the expense of specificity. In consequence, additional sampling is therefore essential if an AMI is suspected. To improve the specificity of hs-cTnT values for the diagnosis of AMI, it appears crucial to evaluate the relative or absolute change of cTn.

To date, the guidelines recommend troponin kinetics and the kinetic criteria are used to rule in/rule out AMI [16]. However, using relative or absolute changes remains under debate as well as the magnitude of the kinetic change that best discriminates between acute and chronic increases. Several studies demonstrate the superiority of absolute delta change in comparison to relative delta change $[7,8]$. Others propose to use the relative delta change when the values of cTn at admission are $<99$ th percentile, and to use the absolute delta change when the values at baseline are $>99$ th percentile [8]. However, in clinical practice the use of several algorithms can be misleading. In our population, the cut-off $7 \mathrm{ng} / \mathrm{L}$ for absolute delta change as proposed by Reichlin et al., seems to generate false-positive results. Indeed, as regards the absolute delta change values with both assays (see the Results section, Table 3 ) the absolute increase of $7 \mathrm{ng} / \mathrm{L}$ does not seem to be relevant in our population. However, we confirm the superiority of the absolute delta change criterion given the absence of overlap between AMI and non-AMI patients particularly with hs-cTnT assay. The combination of relative and absolute delta changes improves accuracy and provides an additional benefit reducing greatly false-positive results with both assays. In addition, with this algorithm we do not observe any false-negative results confirming the same specificity of both assays.

Our study includes several limitations. The number of subjects with blood samples on admission and $3 \mathrm{~h}$ later is small with only few patients 
in each clinical category, including for the AMI group ( $n=8)$. However, because this study includes a population of consecutive patients presenting with non-traumatic chest symptoms, a significant proportion (87\%) has non-AMI suggesting inappropriate investigations. Another limitation is the lack of information on the time between symptom onset and cTn sampling on admission. This variable is of great importance, particularly in patients with an early management or with delayed presentation after symptom onset. Indeed, the time interval for sampling should be consistent with the clinical context on admission to allow discrimination of acute from chronic reasons for the cTn increase.

\section{Conclusion}

Our results indicate the acceptable analytical performances of cTnT AQT90 FLEX ${ }^{\circledR}$ on AQT90® instrument when compared to the hs-TnT and as regards clinical classification of a non-selected population. The results with the $A Q T 90 \AA$ instrument highlight that the cTnT assay is less sensitive than the Roche hs-cTnT assay. However, negative values on admission should be interpreted with caution and do not exclude the diagnosis of AMI. On the contrary, the kinetic change is of great importance, since in our study it allows to assert unambiguously the diagnosis of AMI similarly with both assays. The determination of optimal delta criteria in clinical studies particularly with POC instrument requires additional studies.

\section{Conflicts of interest}

The authors declare no financial or competing interests.

\section{Acknowledgments}

The cTnT reagents used in this study were provided by Radiometer (Neuilly-Plaisance, France).

\section{References}

[1] Hamm CW, Bassand JP, Agewall S, Bax J, Boersma E, Bueno H, et al. ESC Committee for Practice Guidelines. ESC Guidelines for the management of acute coronary syndromes in patients presenting without persistent ST-segment elevation: the task force for the management of acute coronary syndromes (ACS) in patients presenting without persistent ST-segment elevation of the European Society of Cardiology (ESC). Eur Heart J 2011;32(23):2999-3054.
2] Storrow AB, Apple FS, Wu AH, Jesse R, Francis G, Christenson RH. Use of cardiac biomarkers for acute coronary syndromes. In: Nichols JH, editor. National Academy of Clinical Biochemistry. Laboratory Medicine Practice Guidelines; Evidence-Based Practice for Point-of-Care Testing. Washington, DC: AACC Press; 2006. p. 13-20 (http://www.aacc. org/SiteCollectionDocuments/NACB/LMPG/POCTLMPG. pdf\#page $=1$. (Accessed February 5, 2012)).

[3] Apple FS, Collinson PO. FCC Task Force on Clinical Applications of Cardiac Biomarkers. Analytical characteristics of high-sensitivity cardiac troponin assays. Clin Chem 2012;58(1):54-61.

[4] Palamalai V, Murakami MM, Apple FS. Diagnostic performance of four point of care cardiac troponin I assays to rule in and rule out acute myocardial infarction. Clin Biochem 2013;46(16-17):1631-5.

[5] Apple FS. A new season for cardiac troponin assays: it's time to keep a scorecard. Clin Chem 2009;55(7):1303-6.

[6] Apple FS, Parvin CA, Buechler KF, Christenson RH, Wu AHB, Jaffe AS. Validation of the 99th percentile cutoff independent of assay imprecision (\%CV) for cardiac troponin monitoring for ruling out myocardial infarction. Clin Chem 2005;51: 2198-200.

[7] Reichlin T, Irfan A, Twerenbold R, Reiter M, Hochholzer W, Burkhalter H, et al. Utility of absolute and relative changes in cardiac troponin concentrations in the early diagnosis of acute myocardial infarction. Circulation 2011;124(2):136-45.

[8] Mueller M, Biener M, Vafaie M, Doerr S, Keller T, Blankenberg S, et al. Absolute and relative kinetic changes of high-sensitivity cardiac troponin $\mathrm{T}$ in acute coronary syndrome and in patients with increased troponin in the absence of acute coronary syndrome. Clin Chem 2012;58(1):209-18.

[9] Irfan A, Reichlin T, Twerenbold R, Meister M, Moehring B, Wildi K, et al. Early diagnosis of myocardial infarction using absolute and relative changes in cardiac troponin concentrations. Am J Med 2013;126(9):781-8.

[10] Lövgren T, Meriö L, Mitrunen K, Mäkinen ML, Mäkelä M, Blomberg K, et al. One-step all-in-one dry reagent immunoassays with fluorescent europium chelate label and time-resolved fluorometry. Clin Chem 1996;42:1196-201.

[11] Apple FS, Collinson PO. IFCC Task Force on Clinical Applications of Cardiac Biomarkers. Analytical characteristics of high-sensitivity cardiac troponin assays. Clin Chem 2012;58(1):54-61.

[12] Dupuy AM, Lozano C, Badiou S, Bargnoux AS, Kuster N, Cristol JP. Biological variability of hs-cardiac troponin T on the Roche Cobas 8000/e602® immunoanalyzer. Clin Chim Acta 2013;425:62-3.

[13] Collinson PO, Heung YM, Gaze D, Boa F, Senior R, Christenson R, et al. Influence of population selection on the 99th percentile reference value for cardiac troponin assays. Clin Chem 2012;58(1):219-25

[14] CLSI. Evaluation of precision performance of clinical chemistry devices; approved guideline. NCCLS Document EP5-A. Wayne (PA): CLSI; 1992.

[15] CLSI. EP17-A, Protocols for determination of limits of detection and limits of quantitation. Wayne, PA: Clinical and Laboratory Standards Institute; 2004.

[16] Thygesen K, Alpert JS, Jaffe AS, Simoons ML, Chaitman BR. White HD; Joint ESC/ ACCF/AHA/WHF Task Force for the Universal Definition of Myocardial Infarction. Third universal definition of myocardial infarction. Circulation 2012;126(16): 2020-35.

[17] Jaffe AS, Apple FS, Morrow DA, Lindahl B, Katus HA. Being rational about (im)precision: a statement from the Biochemistry Subcommittee of the Joint European Society of Cardiology/American College of Cardiology Foundation/American Heart Association/World Heart Federation Task Force for the definition of myocardial infarction. Clin Chem 2010;56(6):941-3. 